\title{
MODELAMIENTO DE LA VOLATILIDAD DEL ÍNDICE GENERAL DE LA BOLSA DE VALORES DE LIMA, PERIODO 2009 - 2011
}

\author{
EXCHANGE LIMA, PERIOD FROM 2009 TO 2011
}

\author{
Rino Nicanor Sotomayor Ruiz ${ }^{1}$ y ${ }^{2}$ Jorge Enrique Castillo Gamarra
}

\begin{abstract}
Resumen
El presente trabajo tiene como objetivo realizar el modelamiento de series de tiempo que presentan como característica principal una fuerte volatilidad, con periodos de calma o agitación, para este fin se emplean los modelos de varianza condicional ARCH y GARCH. Se presentan los modelos, sus propiedades y pruebas estadísticas necesarias para su aplicación. La serie en estudio fue el valor diario del Índice General de la Bolsa de Valores de Lima (IGBVL), periodo $2009-2011$.

Al analizar los resultados de las estimaciones de los modelos que explicarían la volatilidad diaria de la Rentabilidad del Índice General de la Bolsa de Valores de Lima (RIGBVL), periodo 2009 - 2011, se concluyó que el modelo GARCH $(1,1)$ es el adecuado, debido a que a diferencia de los demás modelos tiene el menor valor tanto en el criterio de información de Akaike (AIC) como en el criterio de información de Schwarz (BIC). Previamente se modeló la media de la RIGBVL con el modelo AR (1).
\end{abstract}

Palabras clave: ARCH, GARCH, IGBVL, Volatility.

\begin{abstract}
This paper aims to describe models ARCH and GARCH conditional variance along with their properties and demonstrations. These models are applied to financial time series because the main feature is highly volatile with periods of calm or agitated; this does not allow using the model of traditional time series that assume constant variances. Also considering an application as a study variable daily value of General Index of the Lima Stock Exchange (General Index) period from 2009 to 2011.

In analyzing the results of the estimates of the models that explain the daily volatility of the Profitability of General Index of the Lima Stock Exchange (RIGBVL) period 2009 - 2011 it was concluded that the GARCH $(1,1)$ model is the appropriate, because unlike the other models have the lowest value in both the Akaike information criterion (AIC) and the Schwarz information criterion (BIC). Previously RIGBVL mean the AR model (1) was modeled.
\end{abstract}

Keys words: Embryonic mortality, Muscovy duck, malpositions, malformations.

\section{Introducción}

En la actualidad estudiar a la información que presentan un comportamiento aparentemente irregular y que influyen de manera directa o indirecta en nuestra sociedad, es de gran interés, como por ejemplo la economía, la cual se encuentra constantemente recabando información. Un tipo información utilizada por la economía es la serie temporal; la cual se define como una secuencia de datos, observaciones o valores, medidos en determinados momentos del tiempo, ordenados cronológicamente y normalmente espaciados entre si y de manera uniforme; con el objetivo de analizar, modelar y predecir el fenómeno de interés. Un caso particular se presenta en las series financieras, las cuales son de principal interés en la actualidad debido a la importancia que implica su comprensión.

Dentro de las características principales que tienen las series financieras se encuentra la volatilidad, el exceso de esta dificulta la planificación financiera y podría significar enormes pérdidas y/o ganancias, de manera que conocer su comportamiento resulta crucial. Al analizar la volatilidad en especial el de las series financieras, estas no se comportan de manera constante por lo que el uso de los modelos de series de tiempo tradicionales no es el adecuado.

\footnotetext{
${ }^{1}$ Dpto Estadística e Informática Facultad Economía y Planificación. E-mail: rino@lamolina.edu.pe

${ }^{2}$ Dpto Estadística e Informática Facultad Economía y Planificación 
Engle (1982) en su estudio para modelar la inflación del Reino Unido desarrolló una nueva clase de procesos llamados modelos $\mathrm{ARCH}$, en los cuales la varianza condicionada a la información pasada no es constante y depende del cuadrado de las innovaciones pasadas, mientras que Bollerslev (1986) generaliza los modelos ARCH al proponer los modelos GARCH en los cuales la varianza condicional depende no solo de los cuadrados de las perturbaciones, como Engle, sino además, de las varianzas condicionales de periodos anteriores, con lo cual mediante los modelos ARCH y GARCH la modelización y predicción de la volatilidad de las series financieras mejora.

El objetivo principal del presente trabajo es: Modelar la volatilidad diaria del Índice General de la Bolsa de Valores de Lima (IGBVL), periodo 2009 -2011, mediante el uso de los modelos de varianza condicional ARCH y GARCH, a partir del objetivo principal se desprenden los siguientes objetivos específicos: Presentar el desarrollo teórico de los modelos de varianza condicional $\mathrm{ARCH}$ y GARCH, realizar el proceso de modelación del valor diario del ÍGBVL, periodo 2009-2011, mediante los modelos de varianza condicional ARCH y GARCH, elegir el modelo al cual se ajuste mejor el valor diario del IGBVL, periodo 2009-2011 y realizar el pronóstico de la volatilidad de la rentabilidad diaria del Índice General de la Bolsa de Valores de Lima (RIGBVL) para los días de la primera semana del mes de Enero del año 2012.

El resto del documento se encuentra estructurado de la siguiente forma: La sección 2 describe los materiales y metodología de los modelos de la heterocedasticidad condicional autoregresiva (ARCH y GARCH); La sección 3 muestra los resultados del proceso de modelación de la serie en estudio así como el análisis de estos; y por último en la sección 4 se presenta las conclusiones.

\section{Materiales y métodos \\ 2.1 Descripción de Variable}

El Índice General de la Bolsa de Valores de Lima (IGBVL) es un indicador que permite establecer comparaciones respecto a los rendimientos alcanzados por los diversos sectores (industria, bancario, agrario, minero, servicios, etc.) participantes en la Bolsa, en un determinado tiempo. Se determina a partir de una cartera formada por las acciones más significativas de la negociación bursátil, seleccionados en base a su frecuencia de negociación, monto negociado y número de operaciones.

\subsection{Población y Muestra}

La población es el total de valores que registró el IGBVL para cada uno de los días, entre los años 2009 -2011, mientras que la muestra es el valor diario del IGBVL, que se obtuvo de los reportes emitidos diariamente por la Bolsa de Valores de Lima durante el periodo 2009 -2011, en conjunto estos valores formaron la serie de tiempo para la parte aplicativa del trabajo.

\subsection{Metodología de la Investigación}

El proyecto es de carácter descriptivo e inferencial, dado que describe el comportamiento del IGBVL y obtiene pronósticos de la volatilidad, así mismo es una investigación de tipo no experimental - transaccional, esto debido a que no se tiene ninguna influencia en los datos y son tomados en un momento determinado.

Para el desarrollo del presente proyecto se partió de la siguiente hipótesis: El valor diario del Índice General de la Bolsa de Valores de Lima, durante el periodo 2009 - 2011 se ajusta a un modelo de varianza condicional GARCH, debido a que no cumple con el supuesto de homocedasticidad. Los procedimientos realizados para el análisis de los datos recabados y por consiguiente el contrastar de la hipótesis planteada se detalla a continuación: Cálculo y análisis descriptivo del rendimiento diario del IGBVL. Modelamiento del rendimiento del IGBVL, mediante los modelos de varianza condicional con el uso del software econométrico EVIEWS 7. Selección del modelo de varianza condicional que mejor ajusta el rendimiento del IGBVL y por último el pronóstico de la volatilidad para los días de la primera semana del mes de Enero del año 2012.

\subsection{Volatilidad}

Medida de riesgo que deriva de los cambios de rentabilidad de los activos financieros (Bonos o Acciones), debido a su sensibilidad a informes o rumores de índole políticos, económicos, monetarios o fiscales. La causa de estos cambios se encuentra en las variaciones de los precios de los activos financieros. La volatilidad se asocia estadísticamente con la desviación o variabilidad del rendimiento en torno a su valor medio, Bahi (2007). Al analizar la volatilidad de un determinado activo financiero, esta presenta un conjunto de características particulares como: Exceso de curtosis, persistencia o conglomerado de volatilidad, salto discontinuo de precio, comportamiento asimétrico y la relación con otros mercados.

\subsection{Criterios de Información}

Los criterios de información se basan en el análisis de máxima verosimilitud y dentro los más utilizados en las series de tiempo están: el Criterio de Información Akaike (AIC) y el Criterio de Información de Schwarz (BIC). El criterio de información de Akaike se define como:

AIC $=\frac{-2}{T} \ln ($ verosimilitud $)+\frac{2}{T} n^{\circ}$ parámetros

donde la función de verosimilitud es evaluada con estimaciones de máxima verosimilitud y $\mathrm{T}$ es el tamaño de la muestra. El criterio de información de Schwarz introduce una penalización aún mayor que el criterio de Akaike para el uso de parámetros a partir de un enfoque bayesiano, este se define como: 
$B I C=\frac{-2}{T} \ln ($ verosimilitud $)+\frac{2}{T} n^{\circ}$ parámetros $* \log \left(n^{\circ}\right.$ observaciones $)$

donde la función de verosimilitud es evaluada con estimaciones de máxima verosimilitud y $\mathrm{T}$ es el tamaño de la muestra. Para ambos criterios de información se determina como mejor modelo aquel del cual se obtuvo el menor valor.

\subsection{Test de ARCH-LM}

El test del multiplicador de Lagrange, definido por Engle (1982), consiste en medir la significancia estadística de los coeficientes de los errores al cuadrado contra sus rezagos mediante una regresión lineal, para probar la heterocedasticidad condicional, conocida como los efectos ARCH. El estadístico de prueba de LM es:

$L M_{a r C h}=T \cdot R^{2}$

donde, $\mathrm{T}$ es el número de observaciones y $R^{2}$ es el coeficiente de determinación de la regresión lineal estimada. Si $L M_{\text {arch }}>X_{(Q)}^{2}(5 \%)$, entonces se rechaza la $H_{0}$, con lo cual existe suficiente evidencia para concluir la presencia de los efectos ARCH en los errores al cuadrado, a un nivel de significación del 5\%.

\subsection{Métodos y Evaluación de Pronósticos}

Dentro de los métodos de pronósticos existen dos métodos muy usados: El método de pronósticos dinámico; el cual realiza pronósticos r-periodos adelante, este método se caracteriza por utilizar el último valor pronosticado disponible para el siguiente pronóstico permitiendo la realización de proyecciones de dos a más periodos hacia adelante y el método de pronostico estático; este método realiza pronósticos solo un periodo adelante. Para entender esto se asume que se está en el momento " $t$ " y se emplean las observaciones para generar el pronóstico que corresponde al momento " $\mathrm{t}+1$ ", una vez realizado esto, para generar el pronóstico " $\mathrm{t}+2$ " se utiliza los datos hasta el periodo " $\mathrm{t}+1$ " (no el valor pronosticado para " $\mathrm{t}+1$ ") y se realiza este procedimiento sucesivamente hasta llegar al periodo de interés.

Para determinar que método de pronóstico es adecuado, se usan los siguientes dos criterios estadísticos: La media absoluta de errores al cuadrado (MAPE); representa el cambio porcentual de los errores por lo que su valor se encontrará entre $[0,1]$, si el MAPE es menor a 1 , indicará que los pronósticos realizados son mejores que los efectuados con el modelo de caminata aleatoria. El coeficiente de desigualdad de Theil; mide las ganancias, en términos de pronósticos, de un modelo determinado respecto a un modelo base, que usualmente es el modelo de camino aleatorio. Si el coeficiente toma un valor menor a uno, significa que el modelo que se está utilizando es mejor que el modelo de caminata aleatoria.

\subsection{ModeloAutoregresivo Condicional Heterocedástico (ARCH)}

Los modelos ARCH tienen su origen en un artículo de Engle (1982), en el que se pretende obtener una predicción adecuada para la inflación en el Reino Unido, sujeta a una fuerte volatilidad y con periodos de especial calma o agitación, De Arce (1998). Los modelos ARCH satisfacen dos características básicas: La serie $y_{t}$ es serialmente incorrelacionada pero dependiente y la dependencia de esta se describe mediante una función cuadrática de sus retardos, Zarraga (2011).

El proceso $\mathrm{ARCH}(\mathrm{q})$ viene definido por la extensión:

$$
\begin{aligned}
& y_{t}=\varepsilon_{t} \boldsymbol{h}_{t} y_{t}=\varepsilon_{t} \boldsymbol{h}_{t} \\
& \boldsymbol{h}_{t}^{\mathbf{2}}=\alpha_{\mathbf{0}}+\sum_{i=1}^{q} \alpha_{i} y_{t-1}^{2} \\
& y_{t} / \Psi_{t-1} \sim N\left(\mathbf{0}, h_{t}^{2}\right) y_{t} / \Psi_{t-1} \sim N\left(\mathbf{0}, h_{t}^{2}\right)
\end{aligned}
$$

donde, "q" es el número de retardos de valores pasados $y_{t}$ al cuadrado que se utilizarán para modelar la varianza condicional; $\varepsilon_{t} \sim N I D(0,1)$ y $\boldsymbol{h}_{t}$, son dos procesos estacionarios independientes y $y_{t}$ pueden ser las perturbaciones de un modelo ARMA; $\alpha_{0}$ y $\alpha_{i}$ donde $\mathrm{i}=1, \ldots, \mathrm{q}$ son los parámetros donde la suma de estos debe ser menor a la unidad para que se cumpla la condición de estacionariedad en media.

\subsubsection{Estimación del Modelo ARCH (q)}

Rodríguez (2001), sea el proceso descrito en las ecuaciones (5) y (6). Cada observación $y_{t}$ tiene una distribución condicional normal independiente de las demás, luego el logaritmo de la función de verosimilitud condicional es la suma del logaritmo de la verosimilitud de cada una de las T observaciones muestrales:

$L=\sum_{t=1}^{T} \frac{l_{t}}{T}=\frac{1}{2 T} \sum_{t=1}^{T}\left(\ln h_{t}^{2}+\frac{y_{t}^{\mathbf{2}}}{\boldsymbol{h}_{t}^{\mathbf{2}}}\right)$

El logaritmo neperiano de la contribución del período ${ }^{t}$ a la función de verosimilitud es $\mathbf{l}_{\mathrm{t}} \mathrm{y}$ sus derivadas primera y segunda respecto a $\alpha$ son:

$$
\begin{aligned}
& \frac{\partial l_{t}}{\partial \alpha}=\frac{\mathbf{1}}{2 \boldsymbol{h}_{t}^{2}} \frac{\partial \boldsymbol{h}_{t}^{2}}{\partial \alpha\left(\frac{y_{t}^{2}}{h_{t}^{2}}-1\right)} \\
& \frac{\partial^{2} l_{t}}{\partial \alpha \partial \alpha^{\prime}}=\frac{1}{2\left(\boldsymbol{h}_{t}^{2}\right)^{2}} \frac{\partial \boldsymbol{h}_{t}^{2}}{\partial \alpha} \frac{\partial_{\boldsymbol{h}_{t}^{2}}^{2}}{\partial \alpha^{2}} \frac{y_{t}^{2}}{\boldsymbol{h}_{t}^{2}}+\frac{\left(\frac{y_{t}^{2}}{h_{t}^{2}}-\mathbf{1}\right) \partial}{\partial \alpha^{\prime}} \frac{\mathbf{1}}{2 \boldsymbol{h}_{t}^{2}} \frac{\boldsymbol{h}_{t}^{2}}{\alpha}
\end{aligned}
$$


el gradiente es un vector $(\mathrm{q}+1) \times 1$ y la matriz hessiana es simétrica de orden $(\mathrm{q}+1) \times(\mathrm{q}+1)$.

La estructura ARCH introduce dependencia entre las distribuciones de probabilidad de $\mathbf{y}_{\mathbf{t}}$ correspondientes a periodos sucesivos de tiempo, por lo que tiene sentido definir la matriz de información como la esperanza condicional del hessiano de la función de verosimilitud, cambiada de signo. Además, como $\mathbf{E}_{\mathrm{t}-1}\left(\frac{\mathbf{y}_{\mathrm{t}}^{2}}{\mathbf{h}_{\mathrm{t}}^{2}}\right)=\mathbf{1}$ la matriz de información acaba siendo:

$\vartheta_{\alpha \alpha}=-\sum_{t} E_{t-1}\left(\frac{\partial^{2} l_{t}}{\partial \alpha \partial \alpha^{\prime}}\right)=\sum_{t} \frac{1}{2\left(h_{t}^{2}\right)^{2}} \frac{\partial^{2}}{\partial \alpha} \frac{\partial h_{t}^{2}}{\partial \alpha^{\prime}}$

Ahora consideremos que $\boldsymbol{h}_{t}^{\mathbf{2}}$ es una función lineal como en (5):

$\boldsymbol{h}_{t}^{\mathbf{2}}=\alpha_{0}+\sum_{i=1}^{q} \alpha_{i} y_{t-1}^{\mathbf{z}}=z_{t}^{\prime} \alpha$

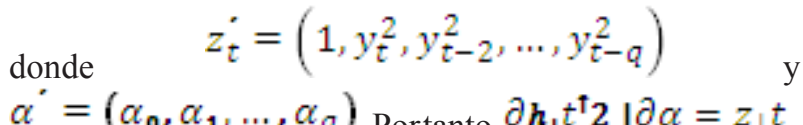

$\frac{\partial \mathrm{y}}{\partial \alpha}=\sum_{t} \frac{\partial l_{t}}{\partial \alpha}=\sum_{t} \frac{1}{2 h_{t}^{2}} z_{t}\left(\frac{y_{t}^{2}}{h_{t}^{2}}-1\right)$

mientras que (10) quedaría como sigue:

$\vartheta_{\alpha \alpha}=\frac{1}{2} \sum_{t} \frac{Z_{t} Z_{t}}{h_{t}^{4}}$

Expresiones que se utilizan en un algoritmo iterativo tipo scoring para obtener el estimador MV de los parámetros del modelo. Este algoritmo es:

$\hat{\alpha}_{i+1}=\hat{\alpha}_{i}+\left(\widetilde{Z}^{\prime} \tilde{z}\right)^{-1} \widetilde{Z}^{\prime} w$

Como criterio de convergencia se utiliza generalmente el estadístico G:

$G=\left(\frac{\partial L}{\partial \alpha^{\prime}}\right)\left(\frac{\partial^{2} l_{t}}{\partial \alpha \partial \alpha^{\prime}}\right)^{-1}\left(\frac{\partial L}{\partial \alpha}\right)$

que se distribuye como $X_{q}^{2}$. Si el estadístico toma un valor superior al de las tablas, se interpreta que el cambio en la estimación es grande, y se continúa iterando. En caso contrario, se detiene el proceso y se conserva el último valor obtenido.

\subsection{Modelo de Heterocedasticidad Condicional Autoregresivo Generalizado (GARCH)}

GARCH es la ampliación del modelo ARCH, que realizó Bollerslev (1986) para los órdenes (p,q). El modelo $\mathrm{ARCH}(\mathrm{q})$ que antes se presentaba, puede mostrar ciertas dificultades de estimación cuando se aplica a estructuras dinámicas en los cuadrados de las series. El modelo GARCH (p,q) se puede escribir como:

$$
\varepsilon_{t}=\eta_{t} h_{t} \varepsilon_{t}=\eta_{t} h_{t}
$$

$h_{\downarrow} t^{\dagger} 2=\alpha_{\downarrow} 0+\Sigma_{\downarrow}(i=1)^{\dagger} q \overline{\bar{z}}-\alpha_{\downarrow} i \varepsilon_{\downarrow}(t-1)^{\dagger} 2 z+\Sigma_{\downarrow}(j=1)^{\dagger} p \overline{\bar{w}}-\beta_{\downarrow} j h_{\downarrow} t^{\dagger} 2||, \varepsilon_{\downarrow} t \mid \Psi_{\downarrow}(t-1) \sim N\left(0, h_{\downarrow} t^{\dagger} 2\right)$

con lo cual, el modelo ARCH (q) anterior no sería más que un caso concreto de este (aquél en el que todos los parámetros $\beta_{j}$ son igual a cero). El modelo GARCH $(1,1)$ tiene las siguientes características: $\eta_{t}$ es proceso estocástico estacionario idénticamente distribuido con media cero y desviación típica igual a uno; los parámetros $\alpha_{0} \geq 0$ y $\alpha_{i}, \beta_{j} \geq 0$ con $\mathrm{i}=1 \ldots \mathrm{q}, \mathrm{y} \mathrm{j}=1 \ldots \mathrm{p}$, además, para cumplirse la condición de estacionariedad en media, la suma de todos los parámetros es menor que la unidad y la función de distribución marginal no es conocida, pero se pueden calcular los primeros momentos y definir el proceso respecto a su media y varianza, De Arce (1998).

\subsubsection{Estimación del Modelo GARCH $(p, q)$ de Regresión}

El modelo se puede definir, en forma esquemática, como:

$\varepsilon_{\downarrow} t=y_{\downarrow} t-x_{\downarrow} t^{\uparrow} \cdot b, \varepsilon_{\downarrow} t \mid \Psi_{\downarrow}(t-1) \sim N\left(0, h_{\downarrow} t^{\uparrow} 2\right)$
$\boldsymbol{h}_{t}^{2}=Z_{t}^{\prime} W$

si $\quad z_{t}^{\prime}=\left(1, \varepsilon_{t-1}^{2} ; \varepsilon_{t-2 ; \ldots ;}^{2} ; \varepsilon_{t-q}^{2} ; h_{t-1 ;}^{2} ; h_{t-2}^{2} ; \ldots ; h_{t-p}^{2}\right)$ $w^{\prime}=\left(\alpha_{0}, \alpha_{1}, \ldots, \alpha_{q}, \beta_{1}, \beta_{2}, \ldots, \beta_{p}\right), \quad$ llamamos al vector de parámetros $\theta=\left(b^{\prime}, w^{\prime}\right)$ tal que $\theta \in \Theta$ , siendo $\Theta$ un espacio euclidiano compacto en que existen todos los momentos finitos de $\varepsilon_{t}$. Posteriormente y de cara a la fijación del criterio de convergencia será necesario referirse al verdadero valor de los parámetros, al que llamaremos $\Theta_{0}$. La función del logaritmo máximo verosímil se puede escribir como:

$L_{t}(\theta)=T^{-1} \sum_{t=1}^{T} l_{t}(\theta)$ 
$l_{t}(\theta)=\frac{1}{2} \log \left(h_{t}^{2}\right)-\frac{1}{2} \varepsilon_{t}^{2} h_{t}^{-1}$

al realizar la primera y segunda derivada de $l_{t}(\theta)$ respecto al vector $\mathrm{w}$ de parámetros donde de forma recursiva $\mathrm{y}$ comenzando por obtener una estimación previa para $\boldsymbol{h}_{t}^{\mathbf{2}} \mathrm{y}$ $\varepsilon_{t}^{2}, t \leq \mathbf{0}$, se logra la siguiente expresión de la derivada parcial respecto a los parámetros del modelo GARCH:

$\frac{\partial \boldsymbol{h}_{t}^{2}}{\partial w}=z_{t} \sum_{i=1}^{p} \beta_{j} \frac{\partial \boldsymbol{h}_{t-1}^{2}}{\partial w}$

Del mismo modo se procede para estimar los parámetros del modelo de regresión derivando en función de "b":

$\frac{\partial \boldsymbol{h}_{t}^{2}}{\partial b}=-2 \sum_{j=1}^{q} \alpha_{j} x_{t-j} \varepsilon_{t-j}+\sum_{j=1}^{p} \beta_{j} \frac{\partial \boldsymbol{h}_{t-j}^{2}}{\partial b}$

Para obtener los estimadores de máxima verosimilitud es necesario recurrir a un sistema iterativo. Bollerslev recomienda el método del algoritmo de Berndt, Hall, Hall y Hausman (1974) - BHHH, en el algoritmo llamaremos $\theta^{(i)} \theta^{(i)}$ al vector de parámetros obtenido en la iteración i-ésima. Para calcular el siguiente valor, se aplica la fórmula:

$\theta^{(i+1)}=\theta^{(i)}+\lambda_{i}\left(\sum_{t=1}^{T} \frac{\partial l_{t} \partial l_{t}}{\partial w \partial w^{\prime}}\right)^{-1} \sum_{t=1}^{T} \frac{\partial l_{t}}{\partial \theta}$

donde la derivada del logaritmo de verosimilitud se evalúa para el valor $\theta^{(i)}$ y $\lambda_{i}$ es el valor o criterio de convergencia elegido que marca el diferencial entre dos resultados de estimación para llegar al resultado final.

\section{Resultados}

Para modelar el Índice General de la Bolsa de Valores de Lima, periodo 2009 - 2011 de acuerdo a los objetivos planteados; en primer lugar se modeló la media utilizando los modelos ARIMA bajo la metodología Box \& Jenkins y luego se utilizó los modelos ARCH y GARCH para modelar la varianza.

\subsection{Análisis de Estacionariedad}

Es importante corroborar el cumplimiento de estacionariedad para poder realizar la modelación del IGBVL, para su detección contamos con el test de Raíces Unitarias de Dickey - Fuller Aumentado.
Tabla 1. Test Dickey - Fuller Aumentado para IGBVL.

Null Hypothesis: IGBVL has a unit root

Exogenous: Constant

Lag Length: 0 (Automatic - based on SIC, maxlag=19)

t-Statistic Prob.*

Augmented Dickey-Fuller test statistic-1.943153 0.3125

Test critical values:

$1 \%$ level -3.438831

$5 \%$ level -2.865173

$10 \%$ level -2.568760

*MacKinnon (1996) one-sided p-values.

Se puede observar en el Tabla 1, que el test no permite rechazar la hipótesis nula al $5 \%$ de nivel de significancia, la hipótesis nula es la no estacionariedad de la serie del IGBVL, por lo tanto se concluye que la serie no es estacionaria. Este resultado nos lleva a analizar una serie derivada de la misma, específicamente la rentabilidad diaria del Índice General de la Bolsa de Valores de Lima (RIGBVL). A continuación se presenta el resultado obtenido para determinar si la RIGBVL es una serie estacionaria, que permitirá continuar con el proceso de modelación.

Tabla 2. Test Dickey - Fuller Aumentado para RIGBVL.

Null Hypothesis: RIGBVL has a unit root

Exogenous: Constant

Lag Length: 0 (Automatic - based on SIC, maxlag=19)

t-Statistic Prob.*

Augmented Dickey-Fuller test statistic-25.77534 0.0000

Test critical values:

$5 \%$ level -2.865178

$1 \%$ level -3.438842

$10 \%$ level -2.568763

*MacKinnon (1996) one-sided p-values.

En base al Tabla 2, se observa que el estadístico de prueba de Dickey-Fuller Aumentado en valor absoluto es 25.7753 y el p_value es menor al 5\% de nivel de significancia lo cual conduce a rechazar la hipótesis nula, esto indica que la RIGBVL es estacionaria. Una vez que ya contamos con una serie de tiempo estacionaria podemos continuar con la modelación de la RIGBVL con los modelos ARIMA. De los modelos tentativos para modelar la media de la RIGBVL se consideró como el mejor al modelo AR (1); debido a que el parámetro estimado que se obtiene es significativo. Por lo tanto el modelo se define como:

$$
R I G B V L_{t}=0.064674 * R I G B V L_{t-1}+\varepsilon_{t}
$$

Así mismo se realizó la prueba de autocorrelación "Q" de Ljung Box, el cual determina que los residuos obtenidos con el modelo AR (1) siguen un comportamiento aleatorio, verificando de este modo que la estimación realizada con el modelo es correcta, esto permite realizar el modelado de la volatilidad de la RIGBVL. Antes de continuar con el modelado se presenta los hechos estilizados más resaltantes de la RIGBVL, característica de toda serie financiera. 


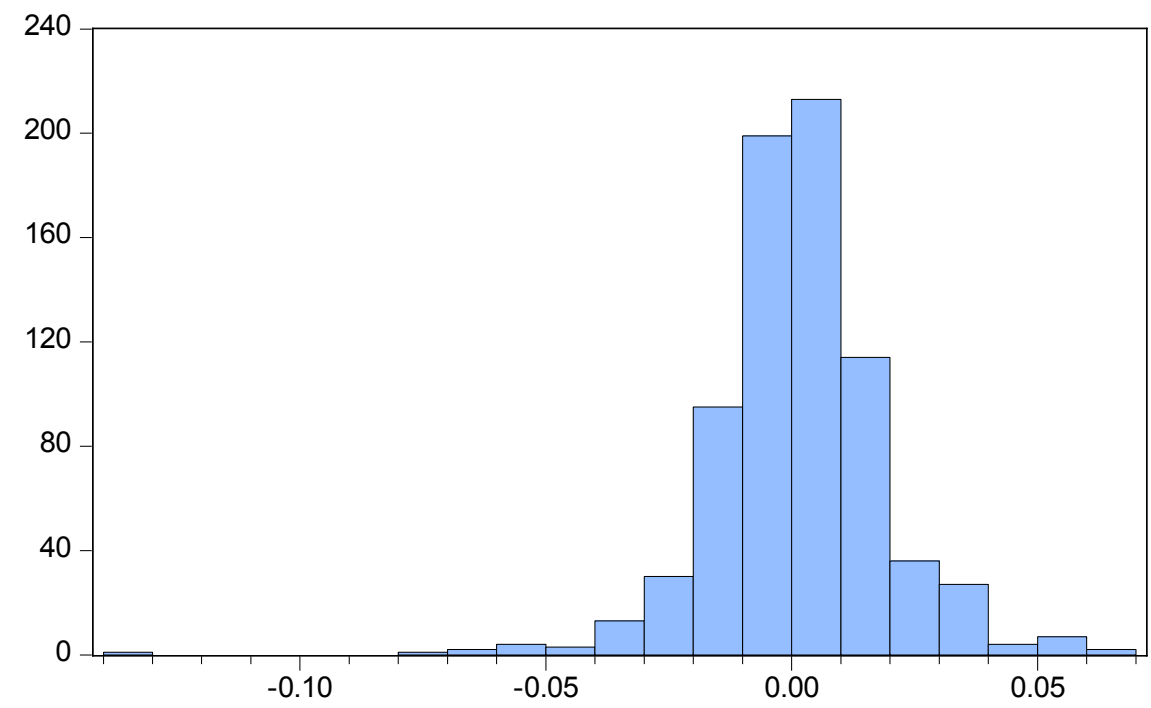

\begin{tabular}{|lc|}
\hline \multicolumn{2}{|l|}{ Series: RIGBVL } \\
Sample 1/05/2009 12/30/2011 \\
Observations & 751 \\
Mean & 0.001301 \\
Median & 0.001074 \\
Maximum & 0.069163 \\
Minimum & -0.132908 \\
Std. Dev. & 0.017466 \\
Skewness & -0.512053 \\
Kurtosis & 9.385433 \\
& \\
Jarque-Bera & 1308.697 \\
Probability & 0.000000 \\
\hline
\end{tabular}

Figura 1. Histograma de la RIGBVL diaria.

En la Figura 1, se observa que la distribución de la rentabilidad diaria de la RIGBVL es leptocúrtica (característica de las series financieras), dado que el valor de Curtosis de la RIGBVL (9.38) es mayor al de una distribución normal (3.00). El p value asociado al estadístico de prueba de Jarque - Bera muestra que la rentabilidad diaria del IGBVL no sigue una distribución normal.

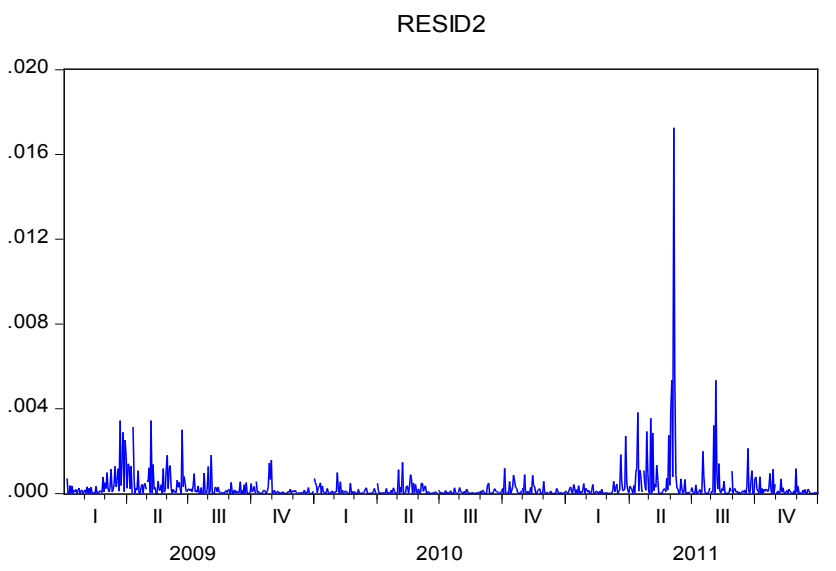

Figura 2. Errores al cuadrado del modelo AR (1) de la RIGBVL.
Otra característica que presentan las series financieras es el agrupamiento de la volatilidad que se puede observar en el Figura 2.

\subsection{Presencia de los Efecto ARCH}

Una vez presentada las características continuamos con la modelación de la RIGBVL, bajo el modelo GARCH. Para determinar la presencia de los efectos $\mathrm{ARCH}$ se realiza el test de ARCH-LM sobre los residuos al cuadrado del modelo estimado AR (1).

Tabla 3. Test ARCH - LM para la RIGBVL.

\begin{tabular}{lrcr}
\hline \multicolumn{4}{l}{ Heteroskedasticity Test: ARCH } \\
\hline F-statistic & 52.90701 & Prob. F(1,747) & 0.0000 \\
Obs*R-squared 49.53995 & Prob. Chi-Square(1) & 0.0000 \\
\hline
\end{tabular}

En base a los resultados del Tabla 3, la probabilidad asociada al estadístico $\mathrm{F}$ de contraste se encuentra en la región de rechazo de la hipótesis nula, es decir, se rechaza la hipótesis de varianza constante y se valida la existencia de heterocedasticidad, en base a esta información se procede a modelar la volatilidad de la RIGBVL por medio de los modelos GARCH.

Tabla 4. Resultados al aplicar los modelos GARCH y ARCH.

\begin{tabular}{|c|c|c|c|c|c|}
\hline Modelo & Parámetros & Coeficientes & Significación & \multicolumn{2}{|c|}{ Estadísticos } \\
\hline \multirow{3}{*}{$\operatorname{GARCH}(1,1)$} & $\bar{C}$ & $1.47 \mathrm{E}-05$ & 0.0006 & $\overline{\mathrm{AIC}}$ & -5.544696 \\
\hline & $\operatorname{RESID}(-1)^{2}$ & 0.200177 & 0 & $\mathrm{BIC}$ & -5.520056 \\
\hline & GARCH(-1) & 0.757024 & 0 & & \\
\hline \multirow{4}{*}{ GARCH $(1,2)$} & $\mathrm{C}$ & $1.62 \mathrm{E}-05$ & 0.0005 & AIC & -5.548545 \\
\hline & $\operatorname{RESID}(-1)^{2}$ & 0.176652 & 0 & $\mathrm{BIC}$ & -5.177744 \\
\hline & GARCH(-1) & 1.099606 & 0 & & \\
\hline & GARCH(-2) & -0.324571 & 0.007 & & \\
\hline \multirow{2}{*}{ ARCH (1) } & $\mathrm{C}$ & 0.000187 & 0 & AIC & -5.375775 \\
\hline & $\operatorname{RESID}(-1)^{2}$ & 0.42347 & 0 & $\mathrm{BIC}$ & -5.357294 \\
\hline
\end{tabular}


De acuerdo a los resultados presentados en el Tabla 4, se considera que el modelo que mejor simula el comportamiento del proceso es el modelo $\operatorname{GARCH}(1,1)$, se llegó a esta decisión dado que al comparar los valores del criterio de información de Akaike (AIC) y el criterio de Schwarz (BIC) de los modelos GARCH $(1,2)$ y ARCH (1), estos son superiores al modelo GARCH $(1,1)$, por lo tanto el modelo es:

$$
\begin{aligned}
& \text { RIGBVL } \\
& \sigma_{t}^{2}=1.47 * 10^{-5}+0.200177 \varepsilon_{t-1}^{2}+0.757024 \sigma_{t-1}^{2}
\end{aligned}
$$

Por último para asegurar que el modelo $\operatorname{GARCH}(1,1)$ es el adecuado se realizó el Test ARCH-LM, el cual indica que los errores al Tabla del modelo son homocedásticos, así mismo se verificó que los errores del modelo estimado no están correlacionados, con estos resultados se concluye el modelo estimado es correcto y se procede a realizar los pronósticos de la media y varianza para los días de la primera semana del mes de enero del 2012.

Tabla 5. Pronósticos primera semana Enero 2012.

\begin{tabular}{lcccc}
\hline & \multicolumn{2}{c}{ Pronóstico } & \multicolumn{2}{c}{ Pronóstico } \\
Fecha & \multicolumn{2}{c}{ Dinámico } & \multicolumn{2}{c}{ Estático } \\
& Media & Varianza & Media & Varianza \\
\hline $02 / 01 / 2012$ & 0.0007789 & $8.774 \mathrm{E}-05$ & 0.0007789 & $8.774 \mathrm{E}-05$ \\
$03 / 01 / 2012$ & 0.0001337 & $9.868 \mathrm{E}-05$ & 0.0009331 & $8.545 \mathrm{E}-05$ \\
$04 / 01 / 2012$ & $2.297 \mathrm{E}-05$ & 0.0001091 & 0.0026759 & 0.0001223 \\
$05 / 01 / 2012$ & $3.944 \mathrm{E}-06$ & 0.0001192 & 0.0004588 & 0.0001073 \\
$06 / 01 / 2012$ & $6.77 \mathrm{E}-07$ & 0.0001288 & $3.358 \mathrm{E}-05$ & $9.593 \mathrm{E}-05$ \\
Coef. Theil & 0.941 & & 0.802 & \\
MAPE & $96.38 \%$ & & $82.97 \%$ & \\
\hline
\end{tabular}

Como se puede observar en el Tabla 5, tanto el método dinámico y estático son adecuados para realizar pronósticos de la RIGBVL con el método $\operatorname{GARCH}(1,1)$, dado que el coeficiente de Theil para ambos métodos es inferior a uno, esto se refuerza con el valor de la media absoluta de errores porcentuales (MAPE) el cual es inferior al $100 \%$ para ambos métodos.

\section{Conclusiones}

El uso de los modelos GARCH es adecuado cuando la serie financiera presenta alta volatilidad, comportamiento asimétrico y no cumple con los supuestos de estacionariedad y normalidad. Además al modelar la media de la serie, los residuos obtenidos no logran captar toda la información, es decir no cumplen con el supuesto de tener una varianza constante en el tiempo.

Los resultados obtenidos al modelar la media y la volatilidad de la rentabilidad diaria del Índice General de la Bolsa de Valores de Lima (RIGBVL) deben tomarse con cautela, debido a que la medición se realizó para un periodo estable posterior a la crisis financiera del año 2008, donde la serie no presento cambios marcados en su comportamiento permitiendo realizar un mejor proceso de modelación.

Por último el estudio de los modelos GARCH se puede complementar con los modelos asimétricos tales como el modelo EGARCH, TGARCH e IGARCH, dado que estos modelos permiten distinguir la magnitud del cambio ante el alza o baja que sufre la serie.

\section{Literatura citada}

Aydemir, A. (1998). "Forecast performance of threshold autoregressive models - a Montecarlo Study", UWO. Department of Economics Working Papers.

Bahi, Claudia (2007). "Modelos de medición de la volatilidad en los mercados de valores: Aplicación al mercado bursátil argentino". Universidad Nacional de Cuyo.

Bollerslev, T. (1986). Generalized Autoregressive Conditional Heteroscedasticity. Journal of Econometrics, 31, pp. $307-327$.

Campbell, J. y Hentschel, L. (1992). “An asimetric model of changing volatility in Stock Returns", Journal of Financial Economics, 31, pp. 281 - 318.

De Arce, Rafael. (1998).’Introducción a los modelos autoregresivos con heterocedasticidad condicional (ARCH)". Universidad Autónoma de Madrid.

Dickey, D. y Fuller, W. (1979). "Distribution of the Estimators for Autoregressive Time Series with a Unit Root", Journal of the American Statistical Association $76,427-431$

Engle, R. F. (1982). Autoregressive Conditional Heteroscedasticity with Estimates of the Variance of United Kingdom Inflation. Econometric, 50, pp. $987-$ 1008.

Figlewski, S. (1997). Forecasting Volatility, Financial Markets, Institutions and Instruments, Volume 6 Number 1, pp. $2-87$.

Greene, W. (1999). Análisis Econométrico, 3era Ed., Pearson Educación, Madrid, 1999.

Gujarati, D. (2010). Econometría, 5ta Ed., McGrawHill/Interamericana Editores, México D. F., 2010.

Mandelbrot, M. (1963). The variation of certain speculative prices, Journal of Business, 36, 394 - 419.

Márquez, M. (2002). "Modelo Setar Aplicado a la Volatilidad de la Rentabilidad de las Acciones. Algoritmo para su Identificación"(España, Universidad UPC) Cap. II, pp. $11-13$.

Rodríguez, J.(2001).” Una introducción a los modelos de series temporales no lineales", Universidad Rey Juan Carlo, Madrid.

Tsay, R.S. (2002): "Analysis of Financial Time Series, John Wiley \& Sons", New York, Glosten.

Zarraga, A. (2011)."Modelos de Heterocedasticidad Condicionada", Universidad Complutense de Madrid. 